\title{
Numerical insights into the determinants of stent performance for the management of aneurysm with a visceral vessel attached
}

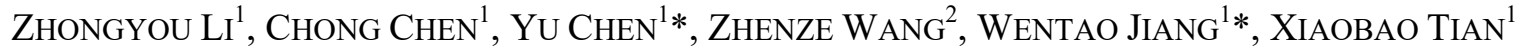 \\ ${ }^{1}$ Department of Applied Mechanics, Sichuan University, Chengdu, China. \\ ${ }^{2}$ Key Laboratory of Rehabilitation Aids Technology and System of the Ministry of Civil Affairs \\ and Beijing Key Laboratory of Rehabilitation Technical Aids for Old-Age Disability, \\ National Research Center for Rehabilitation Technical Aids, Beijing, China.
}

\begin{abstract}
Purpose: As the factors affecting the efficacy of the bare-metal stent in the treatment of aneurysm with a visceral vessel attached were not fully understood, we aimed to discuss the effects of different characteristics of the stent on the hemodynamics and flexibility in the treatment of the aneurysm. Methods: Single-layer (with different strut widths) and multi-layer (with a different number of struts) stent models divided into three porosity groups, with porosities of $72.3,60.5$, and $52.4 \%$, were modeled for a comparison of their hemodynamic isolation and flexibility performance via computational fluid dynamics and finite element methods. Results: The velocity and timeaveraged wall shear stress decreased more noticeably with multi-layer stent interventions. A higher oscillatory shear index and relative residence time occurred at the aneurysmal sac wall after multi-layer stents were employed. Time-averaged wall shear stress on the aneurysmal wall decreased with an increase in the number of struts or a decrease in pore size, but oscillatory shear index and relative residence time increased as the number of struts increased or the pore size decreased. Besides, all stents affect the branch patency slightly. In the bending test, when the porosity exceeded $60.5 \%$, multi-layer stents were more flexible. Conclusion: The number of struts or pore size of stent dominated the isolation in the management of the aneurysm and affected the flexibility significantly when the porosity was below $60.5 \%$. These findings may contribute to the special design of the stent in the treatment of such types of aneurysms.
\end{abstract}

Key words: aneurysm, stent, factors, hemodynamics, flexibility

\section{Introduction}

Although a stent-graft (SG) technique can be applied to most aneurysms due to its isolation, there are still some limitations for aneurysms with visceral vessels attached (such as the pararenal aortic aneurysm), because SG blocks the blood flow of these branches. For this reason, the chimney [1]-[3] and fenestrated [4]-[6] techniques have been approved as two feasible approaches for these aneurysms, but stents for this purpose need to be specially designed owing to individual differences, and it is difficult for clinicians to perform precise surgery.
Therefore, bare-metal stents that overlap each other have gradually received increasing attention in the treatment for such types of aneurysms because of isolation and patency. For example, 61 patients treated by this technique were involved in retrospective research, and the rate of patients freed from rupture was greater than 95\% [7]. Balderi et al. [8] implanted multi-layer stents in five patients with aneurysms, and sac thrombosis and shrinkage were found in four cases (maximal shrinkage rate 57\%) after a two-year follow-up. Factually, the hemodynamic concept of the overlapping bare-metal stents is that blood flow inside the aneurysm declines immediately and pressure returns to normal [9]. Some numerical studies [10]-[12] con-

\footnotetext{
* Corresponding authors: Yu Chen and Wentao Jiang, Department of Applied Mechanics, Sichuan University, Chengdu, 610065 , China. E-mail: yu_chen@scu.edu.cn and scubme@aliyun.com

Received: October 29th, 2020

Accepted for publication: March 19th, 2021
} 
cluded that there was an obvious decrease in velocity and wall shear stress (WSS) inside the sac owing to the isolation of overlapping bare-metal stents, facilitating thrombosis within the aneurysm to reduce the risk of rupture.

Even so, the factors and mechanisms influencing the efficacy of bare-metal stent in the aneurysmal treatment are not fully proven, remaining vague guidance for interventional therapy and specific design of stent, for instance, whether the stent density is the only factor that determines the therapeutic effect? Do other factors including the number of struts, strut width, pore size, et al. affect the performance of the stent? Herein, as lower flexibility leads to a higher probability of damage to the arterial endothelium [13], [14], we modeled single-layer (with different strut widths) and multi-layer (with a different number of struts) stents to analyze the factors affecting the performances for both hemodynamics and flexibility, with the objective of providing guidance for interventional therapy and future designs.

\section{Materials and methods}

\subsection{Computational fluid dynamics (CFD)}

\section{Geometry}

The closed-cell stent configurations (Fig. 1) were established in Solidworks (version 16.0, Dassault Sys-

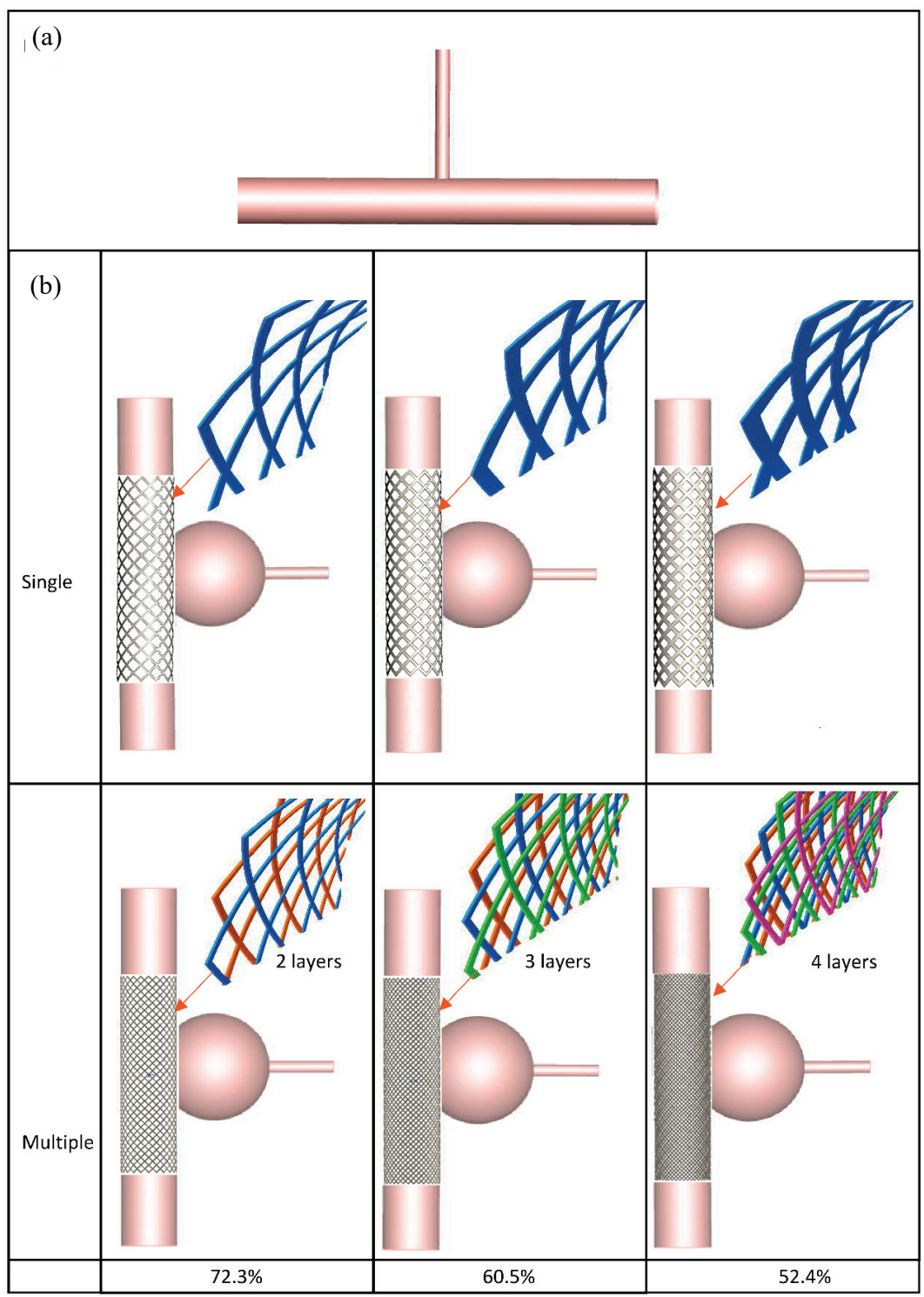

Fig. 1. Geometries. Panel (a) corresponds to the case without an aneurysm. Panel (b) represents three groups of aneurysms after stenting, and the stent porosities are $72.3,60.5$, and $52.4 \%$, respectively. Both single-layer and multi-layer stents were established in each group. Herein, the configurations of multiple stents were referenced by [12] 
(a)

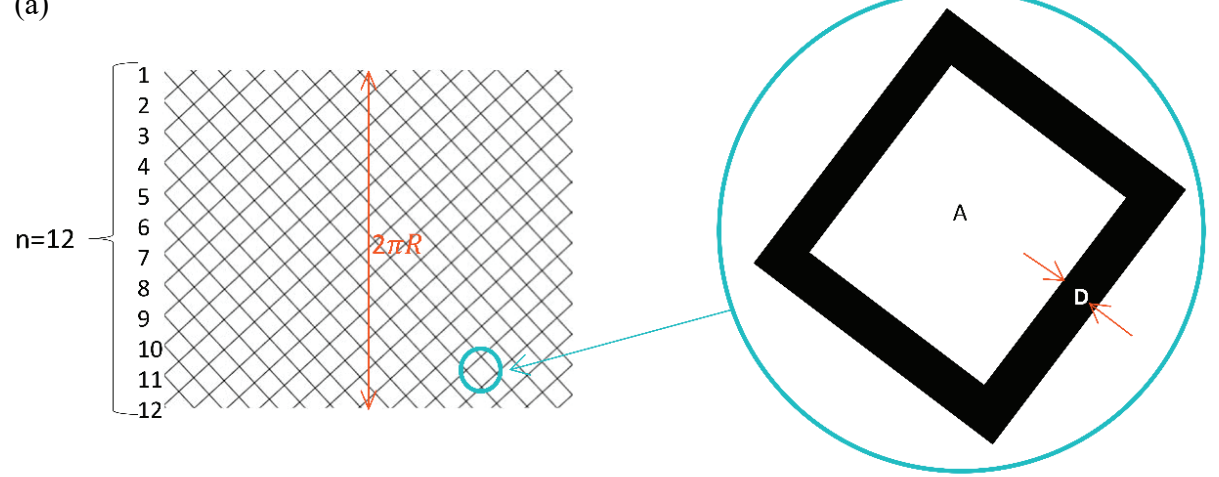

(b)

\begin{tabular}{lllccc}
\hline & & \multicolumn{3}{c}{ Porosity, \% } \\
\cline { 3 - 5 } & & 72.3 & 60.5 & 52.4 \\
\hline Symbol & Parameters & Stent Types & & & \\
\hline \multirow{2}{*}{$\mathrm{n}$} & \multirow{2}{*}{ Number of struts } & Single & 12 & 12 & 12 \\
\cline { 3 - 5 } & & Multiple & 23 & 34 & 45 \\
\hline \multirow{2}{*}{$\mathrm{D}, \mathrm{mm}$} & \multirow{2}{*}{ Width of strut } & Single & 0.544 & 0.816 & 1.088 \\
\cline { 3 - 5 } & Multiple & 0.272 & 0.272 & 0.272 \\
\hline \multirow{2}{*}{$\mathrm{A}, \mathrm{mm}^{2}$} & \multirow{2}{*}{ Pore size } & Single & 9.2515 & 7.5345 & 5.9936 \\
\cline { 3 - 6 } & & Multiple & 2.3506 & 0.8676 & 0.3976 \\
\hline
\end{tabular}

Fig. 2. Basic information about Stents' configuration. Panel (a) is a two-dimensional sketch of stents, where the perimeter of the stent ( $2 \pi R$, where $R$ is the aorta radius) and the number of struts $(n)$ are displayed. For the zoomed-in local element, the pore size (vacant area) and the width of struts are denoted as A and D. Panel (b) lists parameters between two types of stents corresponding to the panel (a)

tèmes SolidWorks Corp) according to Gerberding's patent (US 2014/0180397A1), including a normal case without aneurysm (WA, Fig. 1a) and six aneurysmal models (Fig. 1b). As the porosities (where porosity is the ratio of the total area of holes to the total stent surface area) of the 2-4 layers are 72.3, 60.5 , and $52.4 \%$, respectively, and, as previous studies deemed that the optimal flow modification can be achieved by stents with porosities of $50-70 \%$ [11], [15], [16], both the single-layer (with different strut widths) and multi-layer stents (with a different number of struts [12]) were divided into three porosity groups, with porosities of $72.3,60.5$, and $52.4 \%$. The differences between the two types of stents were designed in Fig. 2. Pore size was defined as:

$$
A=\frac{1}{2} \times\left(\frac{2 \pi R-\sqrt{2} D n}{n-1}\right),
$$

where $R$ represents the aorta diameter, and $D$ and $n$ denote strut width and the number of struts, respectively.

Six fluid domains (vascular models) (Fig. 1) were fabricated by etching. Diameters of 18 and $4 \mathrm{~mm}$ were assigned for aortas and branches, respectively, by considering actual physiological anatomy [17]. True aneurysm [9] with a diameter of $36 \mathrm{~mm}$ [18] was selected to qualitatively analyze the factors influencing the hemodynamic performances of stents in the aneurysmal treatment. Additionally, the intracavitary thrombus was not considered because of its individual differences and complex configuration.

\section{Mesh and governing equations}

ICEM CFD (version 16.0, ANSYS Inc.) was used to generate 1.70 million to 1.98 million mesh elements (Fig. 3), and the "grid adaptation" in Fluent (version 16.0, ANSYS, Inc.) was applied to ensure mesh independence. According to the user's manual of ICEM, as the aspect ratio of each grid was greater than 0.15 , there is no low-quality tetra element. Given that complex stents were modeled, tetrahedron-type elements were used for simulation. Regions such as the arterial wall and stents were refined to ensure the accuracy of the local flow field. The finite volume method in Fluent was adopted for computation, and the control equations used were the time-dependent incompressible Navier-Stokes equations:

$$
\nabla u=0
$$




$$
\rho\left(\frac{\partial u}{\partial t}+u \cdot \nabla u\right)=-\nabla p+\mu \nabla^{2} u,
$$

where $p$ and $u$ refer to pressure and velocity, respectively, and $\rho$ and $\mu$ are the density $\left(1.050 \mathrm{~kg} / \mathrm{m}^{3}\right)$ and dynamic viscosity (0.0035 Pa.s) of blood [19]-[21]. In practice, for diameters of vessels larger than $0.5 \mathrm{~mm}$, the influence of low shear stress on the blood viscosity is relatively small (less than $2 \%$ ), and thus we treated blood as a Newtonian fluid with a constant viscosity [22]. laminar. Transient pressure was given for the aorta (mean pressure $=105.9 \mathrm{mmHg}$ ) and the branch outlet (mean pressure $=100.9 \mathrm{mmHg}$ ), respectively [22]. The values extracted from inflow and outlet pressure waveforms (at $t=0$ ) were given as initial conditions [24]. As the number of elastic fibers decreased and that of collagenous fibers increased during aneurysm growth, the compliance of the aneurysmal wall dropped dramatically [25], so the aneurysmal wall was assumed to be non-slip and rigid-walled [26].

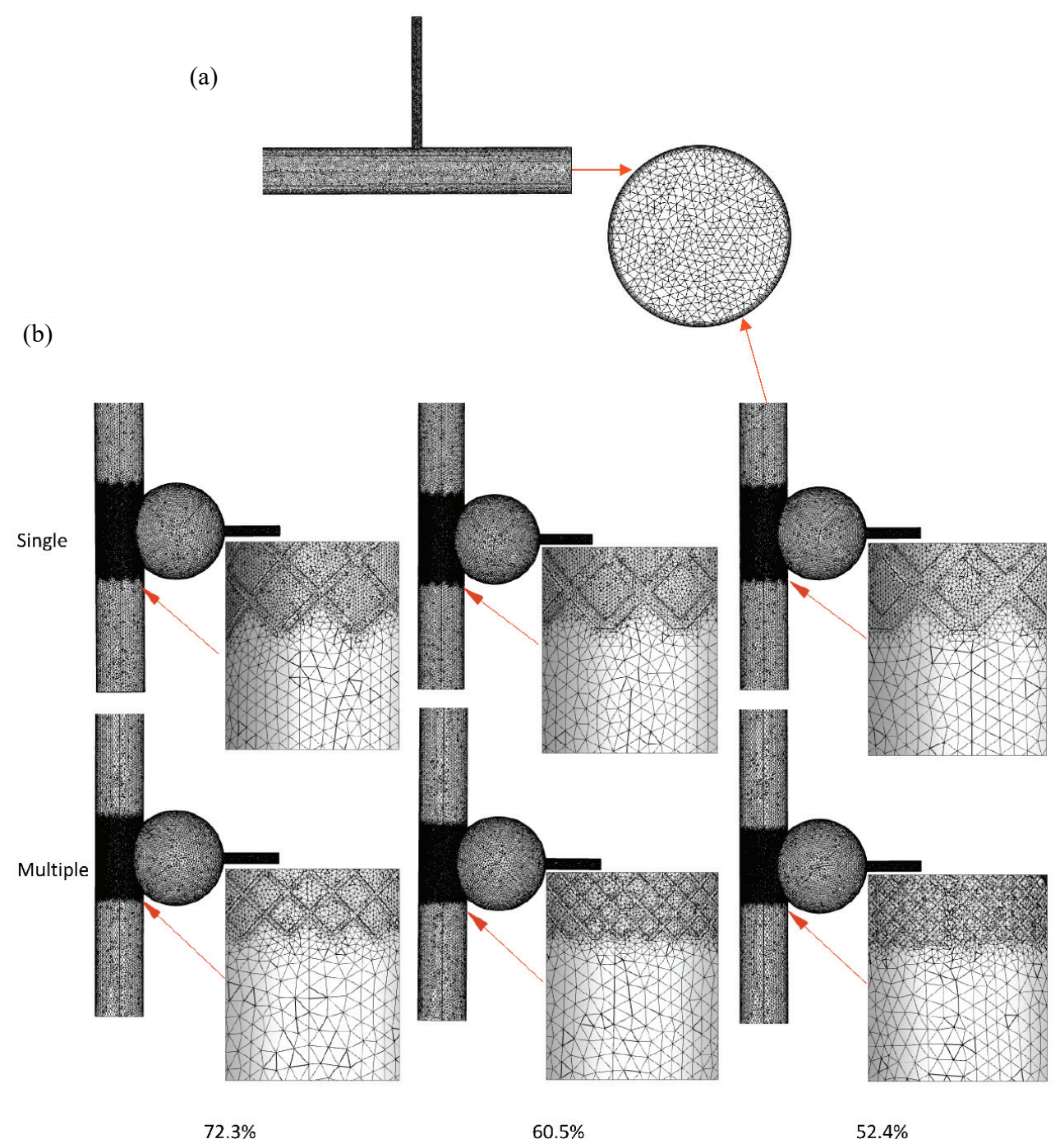

Fig. 3. Mesh. (a) corresponds to the case without aneurysm (WA),

(b) represents the six aneurysmal cases with stents employment.

The refine regions were zoomed in and the boundary layer was indicated

\section{Boundary conditions}

A fully developed pulsatile inflow (mean velocity $=0.24 \mathrm{~m} / \mathrm{s}$ ) was imposed for the inlet boundary condition, and the estimated Reynold's number was 1296 [23]; thus, the blood flow was deemed to be

\section{Hemodynamic parameters}

To qualitatively understand the mechanism of stent's isolation, blood flow field at different time points in the cardiac cycle was extracted; the pressure on the aneurysmal wall was analyzed to evaluate the risk of 
rupture; as thrombosis inside the arterial wall was strongly related to wall shear stress (WSS), oscillatory shear index (OSI) and relative residence time (RRT) [11], we calculated these indicators to compare the hemodynamic performances of different cases. The average WSS of each wall facet during the entire cardiac cycle was represented by the time-averaged wall shear stress (TAWSS):

$$
\mathrm{TAWSS}=\frac{1}{t} \int_{0}^{t}|\mathrm{WSS}(e, t)| \cdot d t,
$$

where $t$ and $e$ are flow time and facet on wall boundary condition, respectively. The directional change of WSS was evaluated by the OSI:

$$
\text { OSI }=\frac{1}{2}\left[1-\left(\frac{\left|\int_{0}^{t} \operatorname{WSS}(e, t) \cdot d t\right|}{\int_{0}^{t}|\operatorname{WSS}(e, t)| \cdot d t}\right)\right] .
$$

The RRT denotes the residence time of the fluid and can be written as:

$$
\mathrm{RRT}=\frac{1}{(1-2 \cdot O S I) \cdot \mathrm{TAWSS}}
$$

Additionally, the blood flow of the visceral vessel in all cases was monitored to assess the effect of stents on the patency of the branch.

\section{Computation procedures}

Equations were solved via the commercial computational fluid dynamics software Fluent (version 16.0, ANSYS, Inc.) based on the finite volume method, and a user-defined function (UDF) was utilized to provide the unsteady inflow and pressure outlet. Method of pressure-velocity coupling is SIMPLEC; Least-Squares Cell-Based, Standard, and Second-Order Upwind schemes were applied to the discretization of gradient, pressure, and momentum, respectively. Residual value corresponded to convergence criteria was set as 0.0001 for each time step $(0.01 \mathrm{~s})$. Four cardiac cycles were expected to be completed to ensure the solution stable, and the results of the fourth cardiac cycle were analyzed [10].

\subsection{Bending test}

\section{Flexibility}

The static finite element method (FEM) was used for the bending test in Abaqus CAE (version 6.13, Das- sault Systèmes SE); tetrahedral elements were selected for simulation. We adopted a cantilever beam model as shown in Fig. 4, and the relative displacement of the free end was used to measure the flexibility of the stent. The material data used for the stents (316L stainless steel) were as follows [27]:

Young's modulus $E=200.000 \mathrm{MPa}$,

Poisson's ratio $V=0.33$,

Yield stress $\sigma=195 \mathrm{MPa}$,

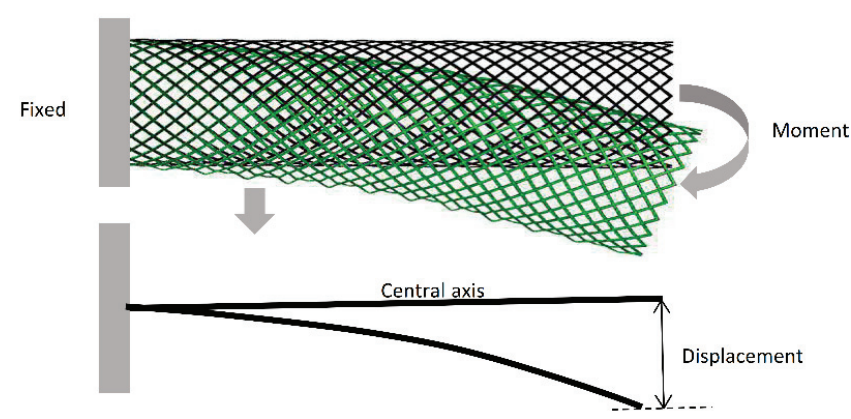

Fig. 4. Cantilever beam model of stent bending. Fixed constraint and coupled moment boundary conditions were each applied to different bilateral ends of the stent. Displacement of the end of the stent is labeled

A fixed constraint boundary condition was exerted on one end of the stent. As locally large deformation and fracture occurred before the entire stent could bend when there was a concentrated load, and thus coupled moment (excluding concentrated load) boundary conditions were incrementally applied to the other end of the stent. The magnitude of the moment applied to the free end of the stents was empirically based on lots of trial calculations. Besides, as internal forces were equal everywhere, the bending was more uniform under moment load conditions.

\section{Results}

\subsection{Pore size}

Depending on Eq. (1), the pore size is strongly correlated with the strut width and the number of stent struts. However, the pore area declines more dramatically with increases in the number of stent struts rather than increases in the strut width (Fig. 5). The pore sizes of single-layer models are 3.9, 8.7, and 15.1 times larger than multiple layers models for groups with porosities of $72.3,60.5$, and $52.4 \%$, respectively. 
Single $(n=12)$

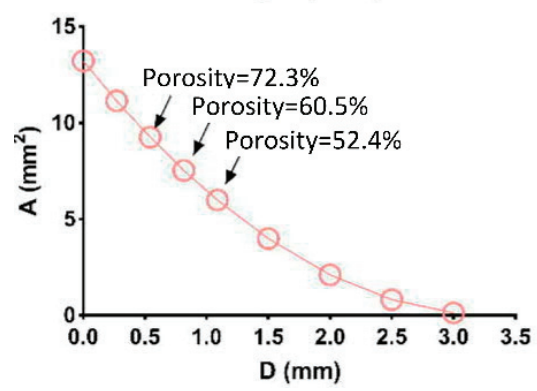

Multiple $(D=0.272 \mathrm{~mm})$

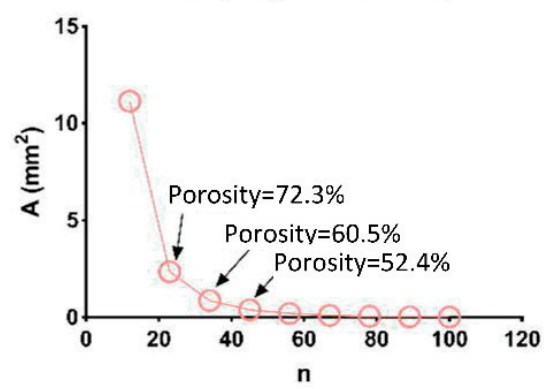

Fig. 5. Correlations between stent pore area and strut width (left) and the number of struts (right). $n$ and $D$ represent strut number and width, respectively

\subsection{Hemodynamic parameters}

\section{Blood flow field}

The flow pattern inside the aneurysm is indicated in Fig. 6, and data of five periods (peak-systole, mid- dle-systole, end-systole, middle-diastole, and end-diastole) were extracted during the cardiac cycle. Disordered flow (uneven flow characterized by large differences in velocity) resulting from stent intervention can be observed at the region near the stent (slice a, Fig. 6), which becomes more laminar at positions further away

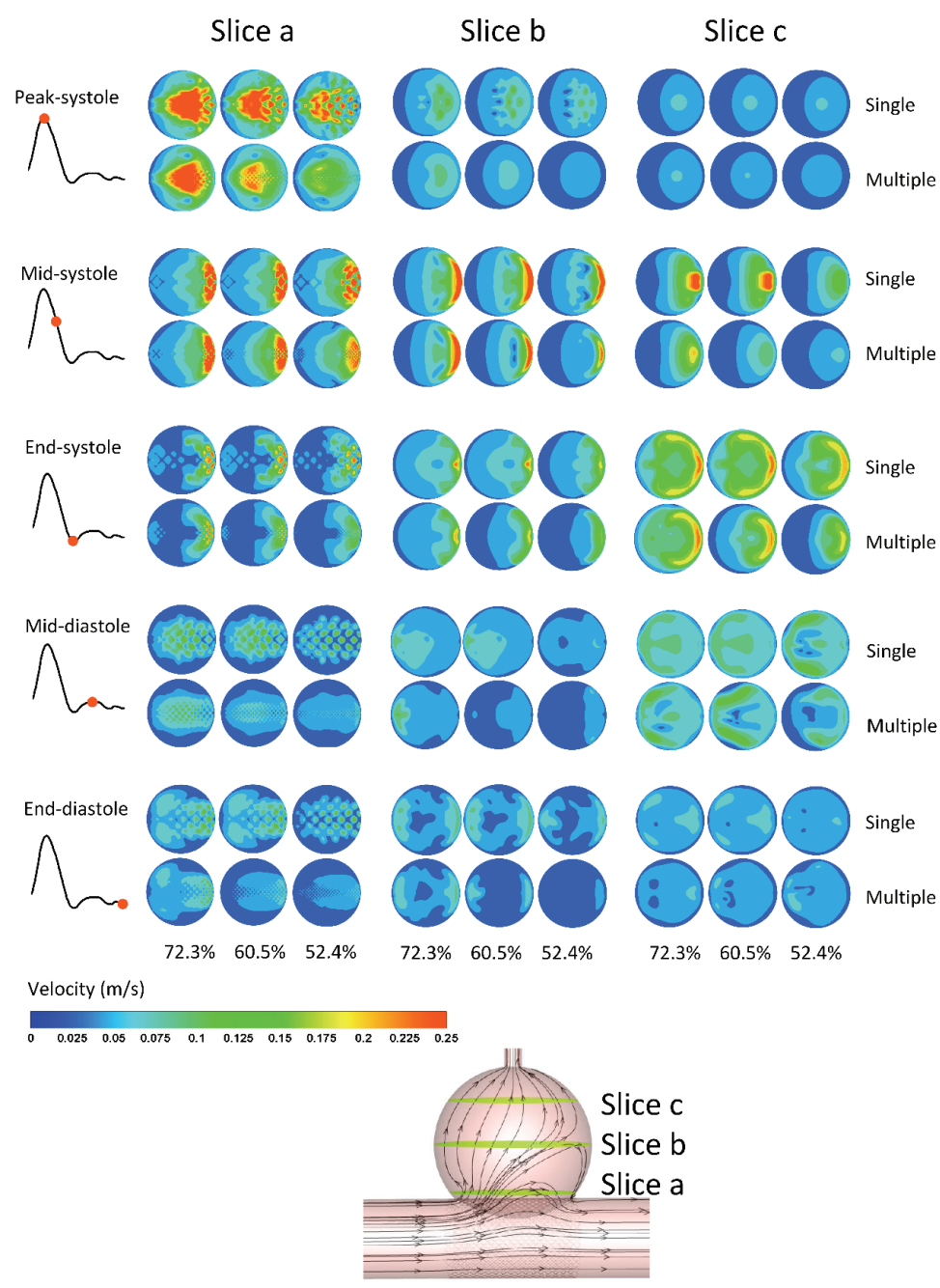

Fig. 6. Blood flow inside the aneurysm through the entire cardiac cycle. Five periods are extracted, including peak-systole, middle-systole, end-systole, middle-diastole, and end-diastole. Slices a, b and c represent the profiles at regions near the stent, middle sac, and near the side branch, respectively. Herein, velocity profiles of multiple stents were referenced by [12] 
from the stent (slices b, c, Fig. 6). In the entire cardiac cycle, although the velocity magnitude decreases with the decreases in porosity for both single-layer and multi-layer cases, it appears that multilayer stents play a more significant role in blocking blood flow and slowing it down, especially in the region near the stents. In addition, the velocity distribution is more uniform for every period of the cardiac cycle with the multi-layer stents implanted, and the peak velocity occurring at the aneurysm downstream is also lower in multi-layer cases than in single-layer cases.

\section{Pressure on aneurysmal wall}

Apart from the vasculopathy of blood vessels themselves, the pressure on the aneurysmal wall contributes greatly to the rupture of aneurysms [28]. However, in both single-layer and multi-layer cases, a slight reduction $(<1 \%)$ in pressure on the aneurysmal wall can be observed with an increase in stents porosity (Fig. 7), distributions on the aneurysmal wall are observed with multi-layer stent implantation (Fig. 8a), and the mean TAWSS (Fig. 8d) decreases by $10 \%(0.354 \mathrm{~Pa}$ vs. $0.318 \mathrm{~Pa}), 25 \%(0.317 \mathrm{~Pa}$ vs. $0.237 \mathrm{~Pa})$, and $40 \%$ $(0.280 \mathrm{~Pa}$ vs. $0.168 \mathrm{~Pa})$ for the cases of $2-4$ layers, respectively. In addition, the mean TAWSS of multilayer cases declines further with an increase in stent porosity than in single-layer cases. For OSI (Fig. 8b), when compared to single-layer cases, the mean OSI on the aneurysmal wall elevates by $137 \%(0.067$ vs. 0.158$), 32 \%$ ( 0.141 vs 0.187$)$, and $46 \%(0.153$ vs. 0.223 ) for the cases of 2-4 layers, respectively. Substantial differences of RRT (Fig. 8c) are observed between single-layer and multi-layer groups, as opposed to the single-layer cases, and the mean RRT increases by $62 \%$ ( 8.67 vs. 14.07$), 148 \%$ (11.32 vs. 28.11$)$, and $367 \%$ ( 15.73 vs. 73.46$)$ for cases of $2-4$ layers, respectively.

According to the linear regression analysis, the number of struts and the pore size has greater effects on the mean TAWSS, OSI, and RRT than strut width (a)

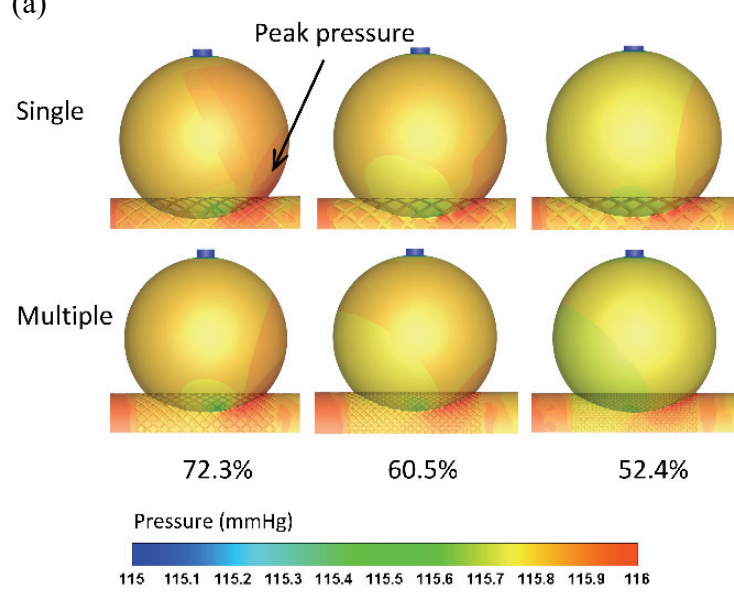

(b)

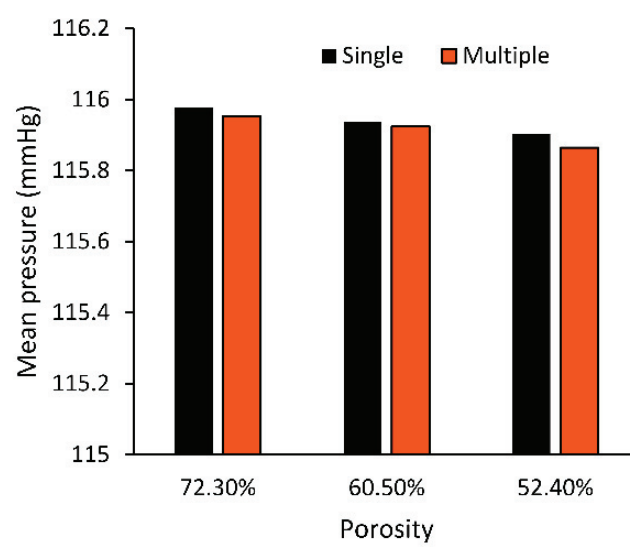

Fig. 7. Pressure distribution on the aneurysmal wall in the peak systole:

(a) is the pressure contour, and the peak pressure on the sac wall is highlighted by an arrow,

(b) is the mean pressure on the sac wall

although lower pressure on the sac wall is observed after multi-layer stent implanting. However, the pressure is distributed more uniformly with four-layer stents intervene.

\section{TAWSS, OSI, and RRT}

Comparisons in terms of TAWSS, OSI, and RRT between single-layer and multi-layer models are shown by both contours and quantitative results (Fig. 8). In comparison with single-layer stents, lower TAWSS
(Fig. 9). The TAWSS decreases with an increasing number of struts and decreasing pore size, while the OSI and RRT increase with an increasing number of struts and reducing pore size.

\section{Patency of the branch}

Generally, it necessary to take the flow rate of the visceral vessel into consideration when aneurysms involve branches, owing to the dysfunction of related organs results from ischemia. Few differences 
are found between the single-layer and multi-layer modes, and the porosity, within the range of 72.3$52.4 \%$, has no effect on the patency of the branch
10). Clearly, the flow rates throughout the entire cardiac cycle are still greater than in the case without an aneurysm.

(a)

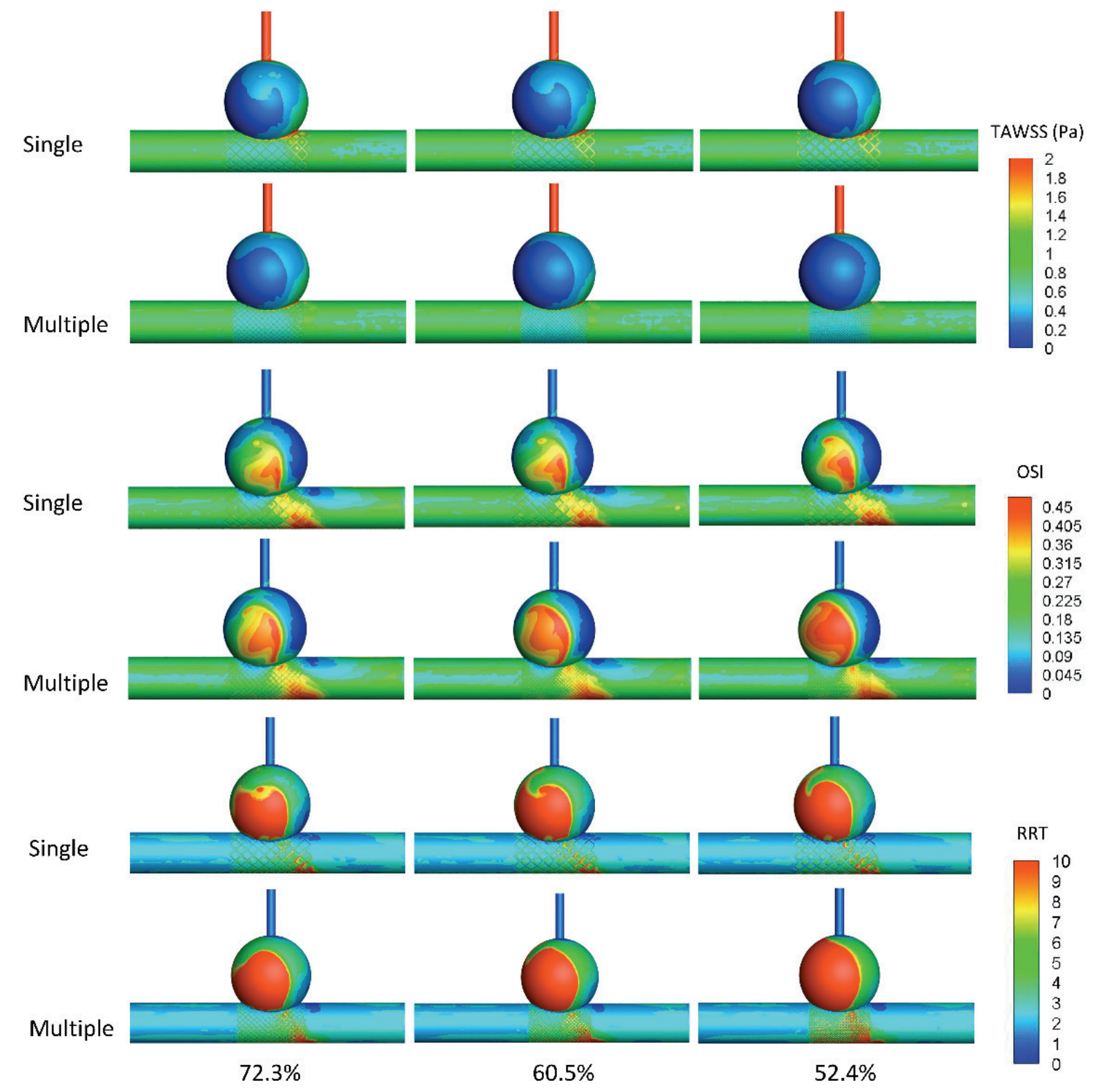

(d)
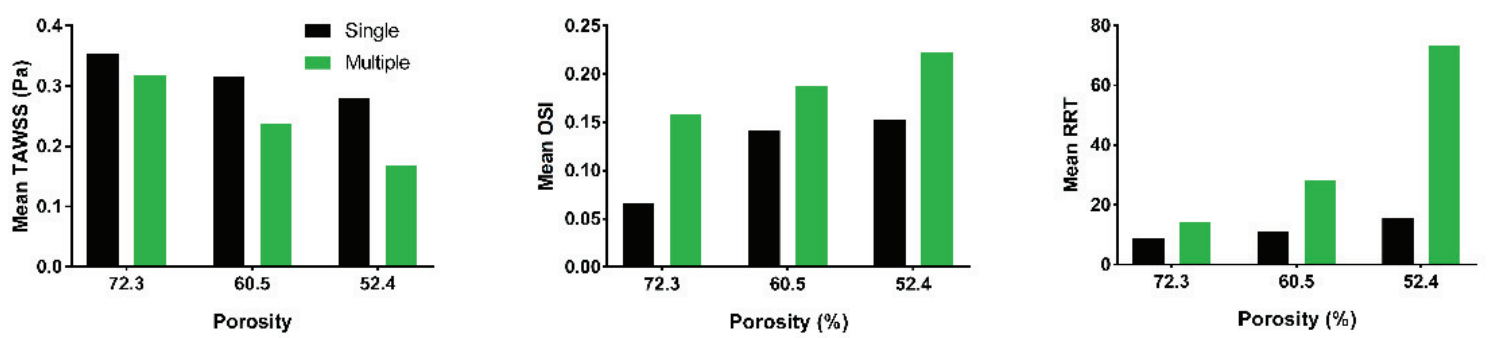

Fig. 8. Distribution of TAWSS, OSI, and RRT: (a), (b) and (c) correspond to contours of TAWSS, OSI, and RRT, respectively; (d) is quantitative data of the mean TAWSS, OSI, and RRT on the sac wall. Herein, these indexes of multiple stents were referenced by [12] 

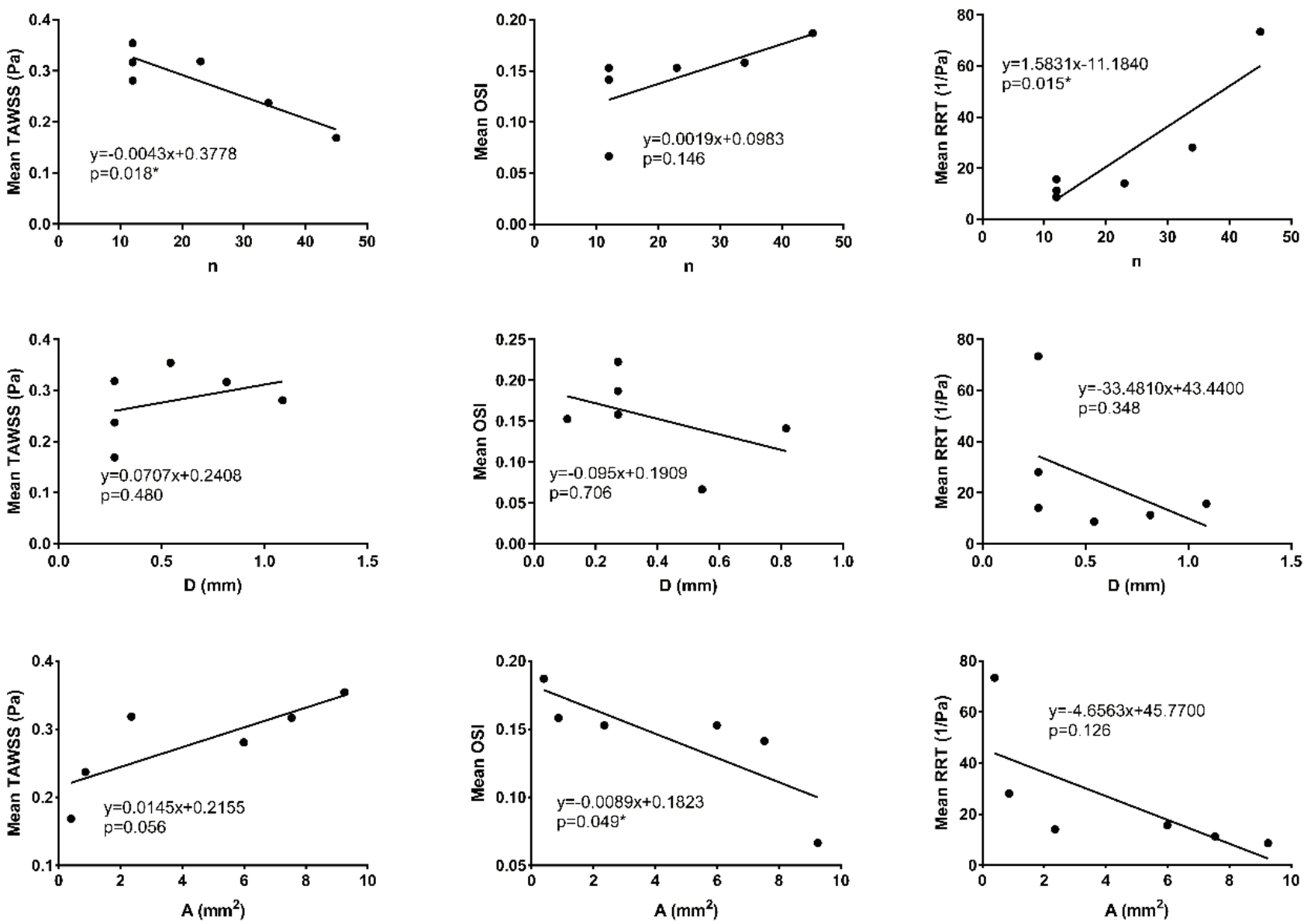

Fig. 9. The effects of the number of struts, strut width and pore size on TAWSS, OSI, and RRT. * represents $p<0.05 . n, D$, and $A$ correspond to the number of struts, strut width, and pore size, respectively

$72.3 \%$

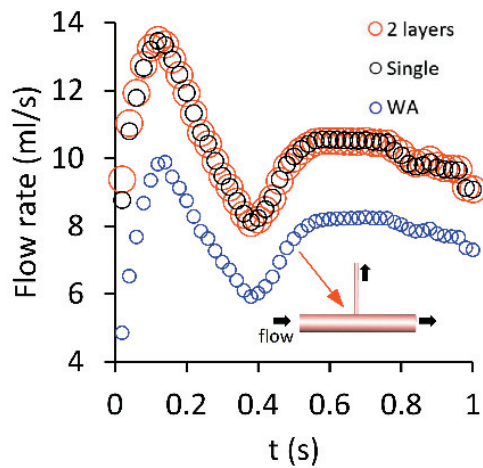

$60.5 \%$

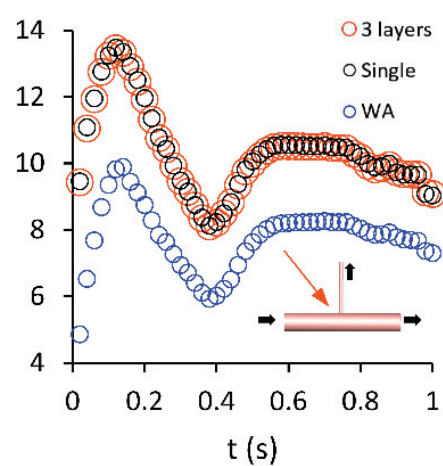

$52.4 \%$

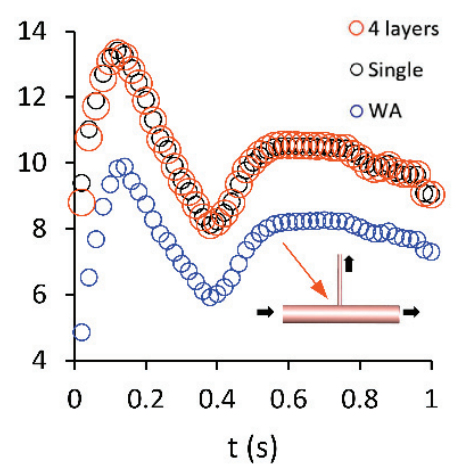

Fig. 10. Flow rate of side branch through the entire cardiac cycle. WA represents the case without aneurysm and is highlighted by a red arrow

\subsection{Bending test}

In the present study, the displacement of the end of the stent was used to evaluate the flexibility of the stent itself (Fig. 11). In all cases, the displacements increase sharply with the bending moment. As porosity increases, the flexibility clearly drops in both the single-layer and multi-layer groups. However, great differences in flexibility are found between singlelayer and multi-layer models with respect to an increase in porosity. Interestingly, single-layer stents are 

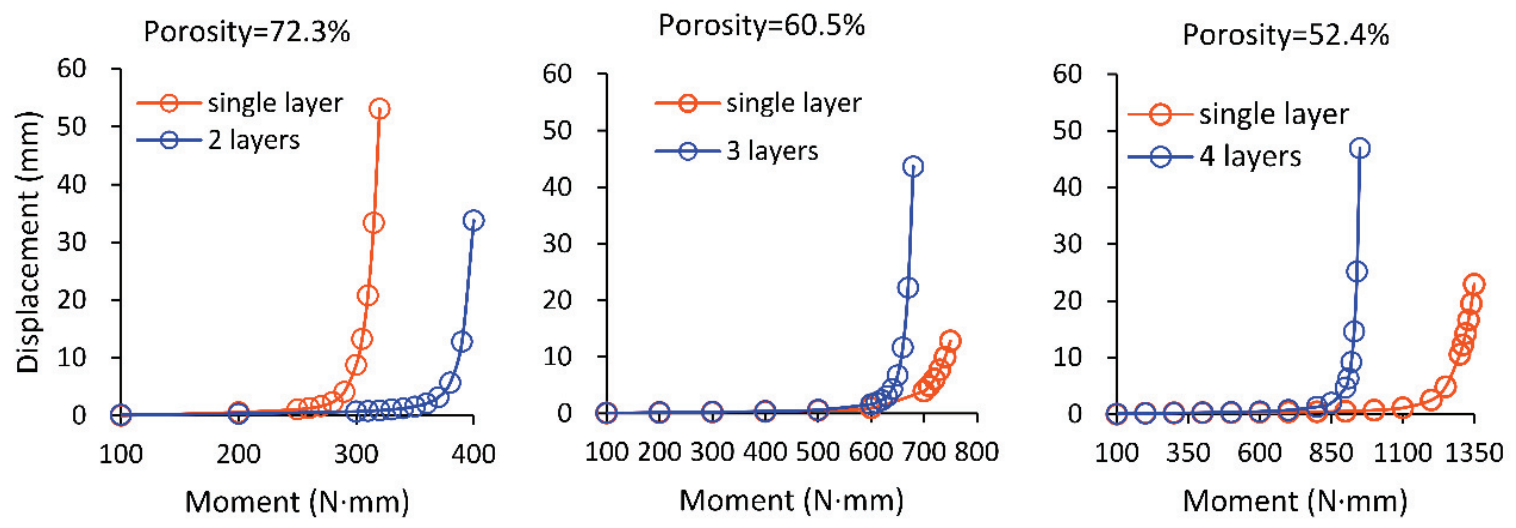

Fig. 11. Relationship between displacement of the stent and its moment

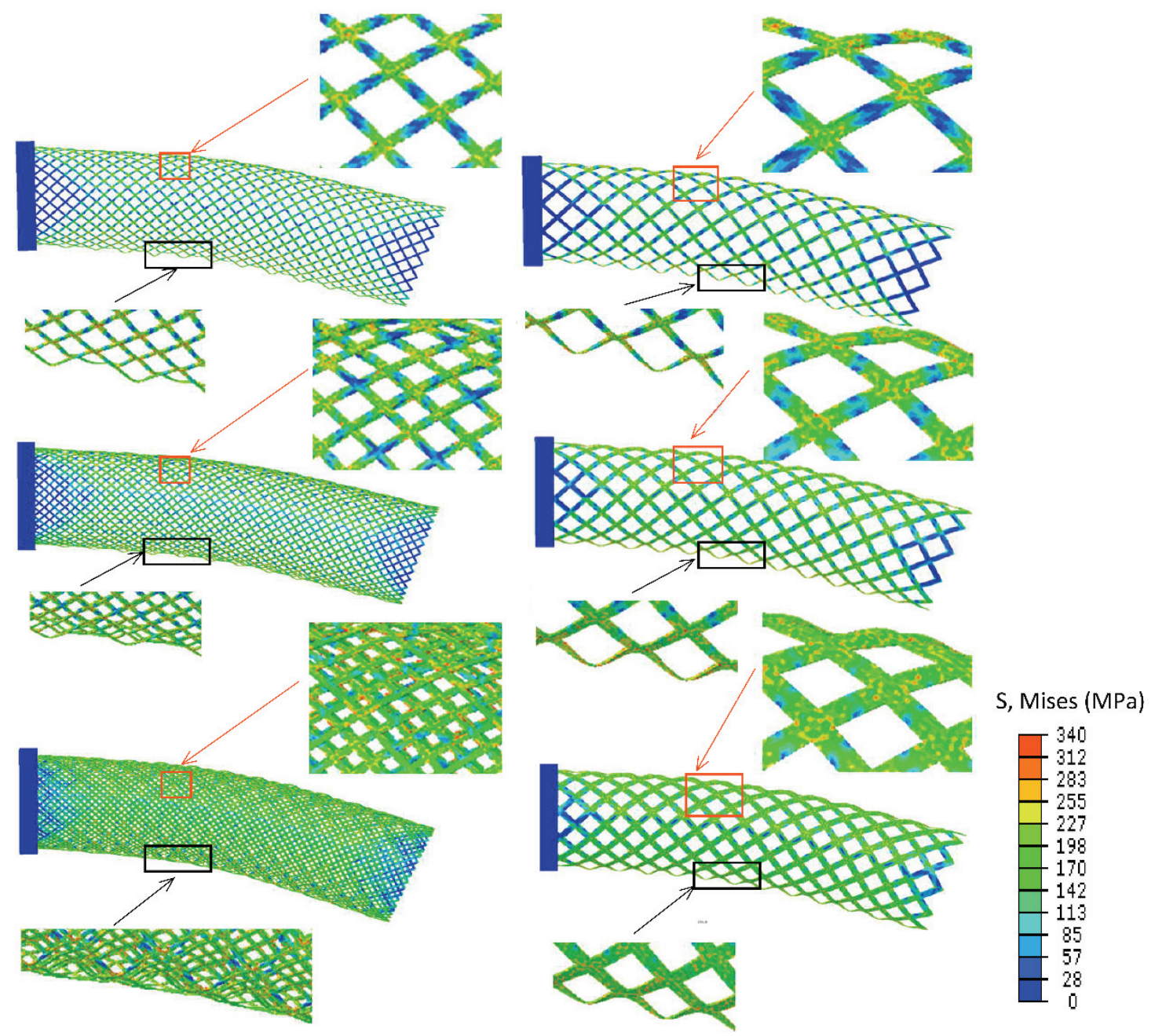

Fig. 12. Deformations and stresses of bending stents. Instabilities are shown in zoomed-in black boxes; stress distribution is shown in zoomed-in red boxes

more flexible than multi-layer stents when the stent porosity is $72.3 \%$, but the opposite results are obtained for the stent porosities of 60.5 and $52.4 \%$.

As opposed to the single design, slighter convexity was observed for multi-layer design while applying a moment to them (Fig. 12). Namely, the strut width has greater effects on the convexity than the number of struts or pore size. Additionally, peak stresses are distributed at the center of the cross in both singlelayer and multi-layer stents. 


\section{Discussion}

Although the efficacy of bare-metal stent has been recognized and confirmed by both numerical [10] and clinical studies [7]-[9], the factors and mechanisms influencing the efficacy of bare-metal stent in the aneurysmal treatment are not entirely known, leading to insufficient guidance for interventional therapy and specific design of the stent.

The principal difference between the configurations of single-layer and multi-layer models is the pore size that was strongly related to the number of struts, and the pore size is an essential factor in determining resistance to blood flow; the smaller the pore, the greater the resistance. The results of the flow features indicated that multi-layer stents play a more significant role in intervening in the blood flow and reducing it; this phenomenon is strongly associated with the pore size of the stent (Darcy's law, $Q=K A h / L$, where $Q$ represents the seepage flow, $K, A, h$, and $L$ refer to seepage coefficient, pore size, head loss, and stent thickness, respectively). Additionally, as the pore size decreases, the flow near the stents declines more obviously. The disappearance of the peak pressure in the aneurysm downstream has a significant connection with the uniform velocity distribution provided by four-layer stents. A uniform distribution of pressure acting on the sac wall can alleviate concentrated stress on the aneurysm, decreasing the risk of rupture [10], [21].

Generally, WSS plays an important role in provoking the release of endothelium nitric oxide (NO); the production of NO decreases as WSS declines [29], [30], which greatly impairs vascular function [31], [32]. Although previous studies reported that the risk of thrombosis increased as WSS declined [33], clinical trials demonstrated that thrombose in the sac helps to form stable layers, which protect the aneurysm from rupture [8], [34]. Our results indicated that TAWSS is lower with the multi-layer stents than with the singlelayer stents, which is strongly correlated with changes in blood velocity [11], [35]; therefore, it is more possible for multi-layer cases to thrombose inside the sac. Moreover, OSI and RRT increased after the multilayer stents were implanted. Therefore, although previous studies [11] have shown that the distribution of low TAWSS, high OSI, and high RRT on the sac wall is beneficial to the growth of the thrombus, our results of OSI and RRT also demonstrated that multi-layer stents have improved isolation, which may help reduce the risk of aneurysmal rupture. In particular, amplified RRT was observed in the multi-layers groups, which would increase the platelet collision rate near the sac wall to thrombose within the aneurysmal wall [36]-[38]. Factually, the linear regression analysis demonstrates that the essential factor for the isolation of stents is the number of struts or pore size (also can be explained by Darcy's law), revealing that multi-layer design plays a more significant role in thrombosis within the sac than single deign.

The patency of the side branch has been confirmed in follow-up studies [7]-[9], but the hemodynamic mechanism is still indistinct. Our results reveal that slight effects of branch patency were caused by stenting despite local intervention because the stent is extremely thin and its resistance is relatively low compared to the arterial wall. Additionally, our results exhibited that stent affects the flow locally; the lower the flow, the lower the reversed flow that was induced by the eddy. On the other hand, local dilation resulting from the development of an aneurysm decreases the resistance of the arterial wall, which is why the branch flow was still above the normal case.

In some cases, the deployment regions of blood vessels are curved; thus, it is necessary to bend the stent to conform to the vessel wall [13]. Along with the material used, stent structure affects flexibility considerably. Our bending test results show that multi-layer stents are more flexible than single-layer stents for porosities exceeding $60.5 \%$ because the mean diameter (half of the difference between the outer and inner diameters) of the whole stents decreases with layer increases, potentially resulting in greater stress and strain when there is an identical moment load. Namely, widening struts have a greater effect on the flexibility of the stent than the increase in the number of struts. However, in this study, when the porosity is $72.3 \%$, the thickness may be the dominant factor in flexibility, but more evidence and explanation are required for these complex configurations.

As convexities may reduce adherence to the arterial wall and even cause damage to the endothelium, the multi-layer design could avoid this phenomenon better because of more struts or small pore sizes. For an explanation of convexity: the deflection increases with the increase in the length of the basic element under the same load condition.

There are still some limitations in the present study. As the goal of this work was to qualitatively analyze the factors influencing the performances of stents, the vessel around the stent was not considered in the bending test. Although the manufacturing process (Nitinol or stainless steel) of the stent may affect the results, we only explored the effects of stent configuration on the flexibility in this work. As the mod- 
els of vessels and aneurysms are greatly simplified to qualitatively discuss the effects of stents' configurations on aneurysmal management, the patient-specific models are to be considered in our next work.

\section{Conclusions}

The number of struts or pore size is a determinant factor of isolation in the management of the aneurysm and has no effect on the patency of the branch. The strut width of the stent affects the stent's flexibility significantly when the porosity exceeds $60.5 \%$. Therefore, instead of widening the strut, increasing the number of struts or reducing the pore size would be a better design to provide better isolation and flexibility. These findings are helpful to guide interventional therapy and design of special stents in the treatment of the aneurysm with a branch attached.

\section{Conflicts of interest}

There are no conflicts of interest in this paper.

\section{Acknowledgement}

This study was supported by Grants-in-Aid from the National Natural Science Foundation of China (No. 11772210), National Key Research and Development Program (No. 2017YFB0702500) and National Natural Science Foundation of China (No. 11272224).

\section{References}

[1] JÉRÉMIE, JAYET, GAEL, et al., A Very Unlucky Intraoperative Event During a Chimney Technique for Juxtarenal Aneurysm Exclusion, Cardiovascular and Interventional Radiology, 2019, 42 (7), 1036-1040.

[2] OvereEm S.P., GOUdEKETting S.R., SCHUURMANN R.C.L. et al., Assessment of changes in stent graft geometry after chimney endovascular aneurysm sealing, Journal of Vascular Surgery, 2019, 70 (6), 1754-1764.

[3] Masayuki, Sugimoto, Giovanni, et al., Impact of renal arterial morphology on fluoroscopy time in chimney endovascular aneurysm repair, Vascular, 2017, 25 (5), 514-519.

[4] Verhoeven E.L.G., Vourliotakis G., Bos W.T.G.J. et al., Fenestrated Stent Grafting for Short-necked and Juxtarenal Abdominal Aortic Aneurysm: An 8-Year Single-centre Experience, European Journal of Vascular and Endovascular Surgery, 2010, 39 (5), 529-536.

[5] Mendes B.C., Greiten L.E., Oderich G.S., Endovascular Repair of a Thoracoabdominal Aortic Aneurysm With a Patient-Specific Fenestrated-Branched Stent-Graft, Journal of Endovascular Therapy, 2017, 1-5.
[6] KoICHI, YuRI, NaOYUKI, et al., A Challenging Treatment for Aortic Arch Aneurysm With Fenestrated Stent Graft, Annals of Thoracic Surgery, 2017, 104 (6), 1915-1922.

[7] Ibrahim W., Spanos K., Gussmann A. et al., Early and midterm outcome of Multilayer Flow Modulator stent for complex aortic aneurysm treatment in Germany, Journal of Vascular Surgery, 2018, 68 (4), 956-964.

[8] Balderi A., Antonietti A., Pedrazzini F. et al., Treatment of Visceral Aneurysm Using Multilayer Stent: Two-Year Follow-Up Results in Five Consecutive Patients, Cardiovascular and Interventional Radiology, 2013, 36 (5), 1256-1261.

[9] Henry M., Polydorou A., Frid N. et al., Treatment of renal artery aneurysm with the multilayer stent, Journal of Endovascular Therapy an Official Journal of the International Society of Endovascular Specialists, 2008, 15 (2), 231-236.

[10] Peng Z., Anqiang S., Fan Z. et al., Hemodynamic study of overlapping bare-metal stents intervention to aortic aneurysm, Journal of Biomechanics, 2014, 47 (14), 3524-3530.

[11] Yeow S.L., Leo H.L., Is Multiple Overlapping Uncovered Stents Technique Suitable for Aortic Aneurysm Repair?, Artificial Organs, 2017, 42 (2), 174-183.

[12] Li Z., Hu L., Chen C., WAng Z. et al., Hemodynamic performance of Multilayer stents in the treatment of Aneurysms with a Branch Attached, Scientific reports, 2019, 9 (1), 1-10.

[13] Alherz A.I., Tanweer O., Flamini V., A numerical framework for the mechanical analysis of dual-layer stents in intracranial aneurysm treatment, Journal of Biomechanics, 2016, 49 (12), 2420-2427.

[14] Auricchio F., Conti M., Ferraro M. et al., Innovative and efficient stent flexibility simulations based on isogeometric analysis, Computer Methods in Applied Mechanics and Engineering, 2015, 295, 347-361.

[15] Augsburger L., Farhat M., Reymond P. et al., Effect of Flow Diverter Porosity on Intraaneurysmal Blood Flow, Clinical Neuroradiology, 2009, 19 (3), 204-214.

[16] LIOU T.M., LI Y.C., Effects of stent porosity on hemodynamics in a sidewall aneurysm model, Journal of Biomechanics, 2008, 41 (6), 1174-1183.

[17] MortaZavinia Z., Arabi S., MehdiZadeh A.R., Numerical investigation of angulation effects in stenosed renal arteries, Journal of Biomedical Physics and Engineering, 2014, $4(1), 1-8$.

[18] YU S.C.M., ChAn W.K., B. T H N., A numerical investigation on the steady and pulsatile flow characteristics in axisymmetric abdominal aortic aneurysm models with some experimental evaluation, Journal of Medical Engineering and Technology, 1999, 23 (6), 228-239.

[19] Li X., LiU X., ZhANG P. et al., Numerical simulation of haemodynamics and low-density lipoprotein transport in the rabbit aorta and their correlation with atherosclerotic plaque thickness, Journal of the Royal Society Interface, 2017, 14 (129), 1-9.

[20] Wada S., Karino T., Theoretical Prediction of Low-Density Lipoproteins Concentration at the Luminal Surface of an Artery with a Multiple layers Bend, Annals of Biomedical Engineering, 2002, 30 (6), 778-791.

[21] Zhongyou Li, Fei YAn et al., Hemodynamics and Oxygen Transport through Pararenal Aortic Aneurysm Treated with Multilayer Stent: Numerical Study, Annals of Vascular Surgery, 2018, 54, 290-297.

[22] Aenis M., Stancampiano P. et al., Modeling of Flow in a Straight Stented and Nonstented Side Wall Aneurysm Model, Journal of Biomechanical Engineering, 1997, 119 (2), 206-212. 
[23] Vignon-Clementel I.E., Figueroa C.A., Jansen K.E. et al., Outflow boundary conditions for three-dimensional finite element modeling of blood flow and pressure in arteries, Computer Methods in Applied Mechanics and Engineering, 2006, 195 (29-32), 3776-3796.

[24] Tomaszewski M., Sybilski K., Malachowski J. et al., Numerical and experimental analysis of balloon angioplasty impact on flow hemodynamics improvement, Acta Bioeng. Biomech., 2020, 22 (3), 1-15.

[25] VORP D.A., Biomechanics of abdominal aortic aneurysm, Journal of Biomechanics, 2007, 40 (9), 1887-1902.

[26] KOPERNIK M., TOKARCZYK P., Development of multi-phase models of blood flow for medium-sized vessels with stenosis, Acta of Bioengineering and Biomechanics, 2019, 21 (2), 63-70.

[27] Walke W., Paszenda Z., Filipiak J., Experimental and numerical biomechanical analysis of vascular stent, Journal of Materials Processing Technology, 2005, 164-165 (none), $1263-1268$.

[28] Dias N.V., IvANCEv K., KölBel T. et al., Intra-aneurysm sac pressure in patients with unchanged AAA diameter after EVAR, European Journal of Vascular and Endovascular Surgery, 2010, 39 (1), 35-41.

[29] Rayan, KhadDaj, Mallat, et al., The vascular endothelium: A regulator of arterial tone and interface for the immune system, Critical reviews in clinical laboratory sciences, 2017, 458-470.

[30] WANG Y.X., LIU H.B., Li P.S. et al., ROS and NO Dynamics in Endothelial Cells Exposed to Exercise-Induced Wall Shear Stress, Cellular and Molecular Bioengineering, 2019, 12 (1), 107-120.

[31] ZHU L., WANG F., YANG H. et al., Low shear stress damages endothelial function through STAT1 in endothelial cells
(ECs), Journal of Physiology and Biochemistry, 2020, 76, 147-157.

[32] JUAn Liu, DA-Jun Hu et al., Attenuated endothelial function is associated with decreased endothelial progenitor cells and nitric oxide in premenopausal diabetic women, Molecular Medicine Reports, 2018, 18 (5), 4666-4674.

[33] Politis A.K., Stavropoulos G.P., Christolis M.N. et al., Numerical modeling of simulated blood flow in idealized composite arterial coronary grafts: Steady state simulations, Journal of Biomechanics, 2007, 40 (5), 1125-1136.

[34] Benjelloun A., HenRy M., GHAnNAM A. et al., Endovascular Treatment of a Tuberculous Thoracoabdominal Aneurysm With the Multilayer Stent, Journal of Endovascular Therapy, 2012, 19 (1), 115-120.

[35] Ouared R., Larrabide I., Brina O. et al., Computational fluid dynamics analysis of flow reduction induced by flowdiverting stents in intracranial aneurysms: a patient-unspecific hemodynamics change perspective, Journal of NeuroInterventional Surgery, 2016, 8 (12), 1288-1293.

[36] Rayz V.L., Boussel L., Ge L. et al., Flow Residence Time and Regions of Intraluminal Thrombus Deposition in Intracranial Aneurysms, Annals of Biomedical Engineering, 2010, 38 (10), 3058-3069.

[37] Ong C.W., Ho P., LeO H.L., Effects of Microporous Stent Graft on the Descending Aortic Aneurysm: A Patient-Specific Computational Fluid Dynamics Study, Artificial Organs, 2016, 40 (11), E230-E240.

[38] Sugiyama S.I., Niizuma K., Nakayama T. et al., Relative residence time prolongation in intracranial aneurysms: A possible association with atherosclerosis, Neurosurgery, 2013, 73 (5), 767-776. 